\title{
Primary subcutaneous extra skeletal Ewing's sarcoma: a case report on a very rare clinical entity
}

\author{
Priya P. Ahire ${ }^{1}$, Pramod D. Nichat ${ }^{1}$, Kashif F. Ansari ${ }^{1}$, Vismaya K. B. ${ }^{1 *}$, Kuldeep Parmar ${ }^{1}$, \\ Aishwarya Mohanraj ${ }^{1}$, Akshay V. Kadam ${ }^{1}$, Subhash Yadav ${ }^{2}$
}

\author{
${ }^{1}$ Department of General Surgery, Grant Government Medical College and J. J. Group of Hospitals, Mumbai, \\ Maharashtra, India \\ ${ }^{2}$ Department of Pathology, TATA Memorial Hospital and Homi Bhabha Research centre, Mumbai, Maharashtra, India
}

Received: 25 July 2021

Revised: 13 August 2021

Accepted: 17 August 2021

\section{*Correspondence:}

Dr. Vismaya K. B.,

E-mail: vismayakb99@gmail.com

Copyright: (C) the author(s), publisher and licensee Medip Academy. This is an open-access article distributed under the terms of the Creative Commons Attribution Non-Commercial License, which permits unrestricted non-commercial use, distribution, and reproduction in any medium, provided the original work is properly cited.

\section{ABSTRACT}

Primary extra skeletal Ewing's sarcoma is a very rare clinical entity. Patients are normally females in the second decade of their life. Clinically it normally presents as a fleshy mass which is mobile, not attached to the underlying structures. Diagnosis is difficult, final diagnosis being made by histopatholoical, immunohistochemistry and cytoskeletal studies combined. Ewing's sarcoma is a tumour which normally affects the bones, and to a much lesser extent $(10 \%)$ the soft tissues. Primary extra skeletal Ewing's sarcoma affecting the back (as in the case we are discussing) is an extremely rare clinical entity with lesser than 50 cases reported worldwide. The entity being so rare doesn't have any much literature for review.

Keywords: Ewing's sarcoma, Extra skeletal, Immunohistochemistry

\section{INTRODUCTION}

Ewing sarcoma is a tumour which normally affects the bones $(90 \%)$, and to lesser extent the soft tissues (10\%). The tumour is very rare, even though it is the second most common bone malignancy found in children. Under the microscope, it is formed by small cells with round hyper chromatic nuclei and a single nucleolus. The cytoplasm is poorly defined, scarce, of clear coloration and with irregular vacuoles resulting from intracellular deposits of glycogen. Immunohistochemically, the cells usually express CD99 and are characterized by a specific chromosomal translocation involving gene EWSR1 in chromosome 22 with an erythroblastosis virus transforming gene, resulting in a fusion oncogene. ${ }^{1,2}$ Since the histology and immune histochemical profile are also relatively nonspecific, many differential diagnoses must be considered, such as Merkel cell carcinoma, cutaneous lymphomas, malignant primitive neuroectodermal tumour or rhabdomyosarcoma. ${ }^{3}$ Patients affected are mostly females with the predilection being 1.9:1, according to Di Giannatale et al. According to Delaplace et al, the female to male ratio is $3: 1$. The median age of diagnosis is 17 years, however in most patients due to difficulty in diagnosing such a rare case, the malignancy would have metastasized (the median time of diagnosis after the onset of symptoms in a case of extra skeletal Ewing's sarcoma is roughly 3-5 months, enough time for a tumour to metastasize and become incurable).

Treatment is mainly surgery. Adjuvant chemotherapy and radiotherapy can also give depending extent of disease. ${ }^{4}$

\section{CASE REPORT}

The patient is a 35-year-old female who initially came to the OPD with complaints of mass on the left trunk for 1 
month. The mass was initially roughly $5 \times 6 \mathrm{~cm}$ in size. The patient then failed to follow up owing to the COVID19 pandemic. The patient finally presented to the OPD again, only now the mass had shown tremendous increase in size, increasing in size from $5 \times 6 \mathrm{~cm}$ to roughly $20 \mathrm{~cm}$ diameter over the left trunk, encroaching on to the lateral border of the spine and possibly involving the erector spinae muscle. FNAC of the mass showed small round cells. After admission, patient underwent thorough pre anesthetic evaluation. She also underwent an MRI of the trunk to look for extent and plane of involvement of the tumour. On MRI it was revealed that the mass is roughly $20 \mathrm{~cm} \times 10 \mathrm{~cm}$ in the deep subcutaneous plane without any deep muscular extension. The erector spinae muscle as well as the spine was uninvolved. The patient underwent surgery to remove the mass, done under GA after proper positioning of the patient (prone position). The patient underwent wide local excision of the, margin taken being $2 \mathrm{~cm}$ circumferentially with rotational flap and suction drain placement under GA. The patient tolerated the procedure well, with no intra-op or immediate post-operative complications

Regarding surgical margins, the published range runs from negative for tumour at the inked margin to $5 \mathrm{~cm}$. According to Kandel et al, the data suggest that patients with clear margins have a better prognosis, but no prospective studies have indicated how wide margins should be..$^{5}$ To preserve functionality, surgery may result in a very close $(<1 \mathrm{~cm})$ or even microscopically positive margins. In this situation, the use of preoperative or postoperative radiation should be considered. Furthermore, no studies described the optimal number of tissue sections required to assess the adequacy of excision nor the appropriate handling of surgical resection specimens.

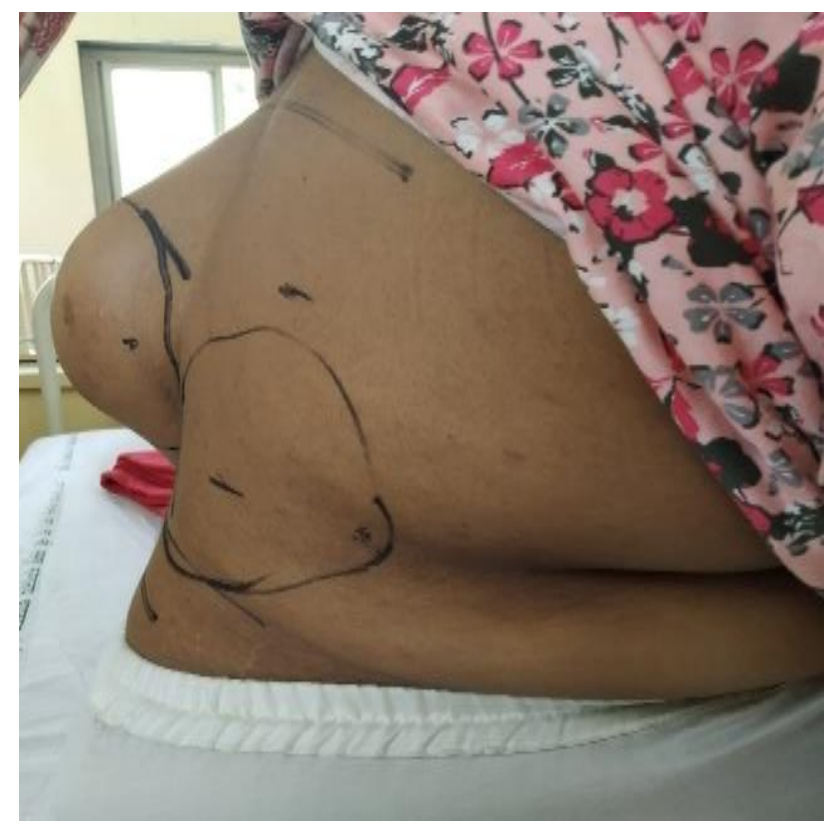

Figure 1: Lateral view of the tumour present on the dorsum.

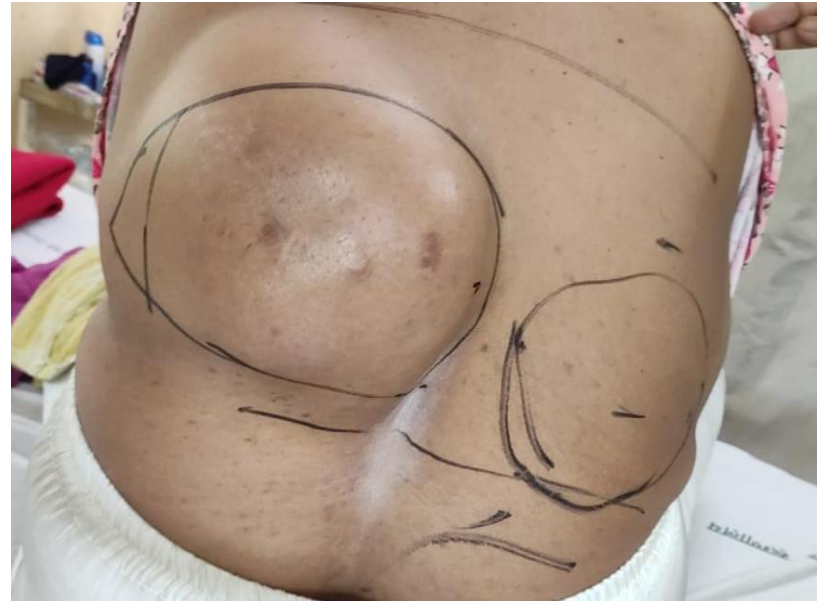

Figure 2: Antero-posterior view of the mass on the dorsum of the back seen encroaching onto the spine.

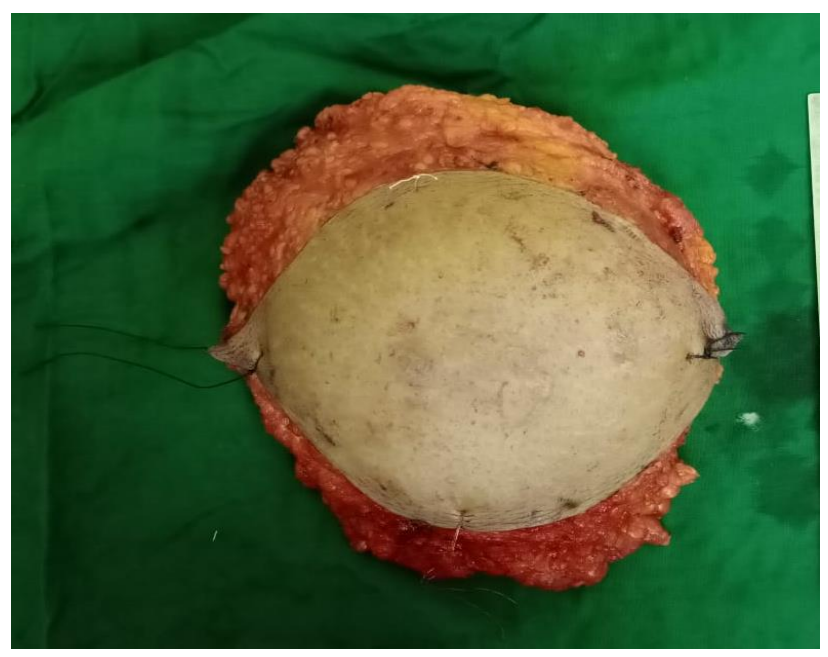

Figure 3: Bird's eye view of the excised mass with scale adjacent to compare.

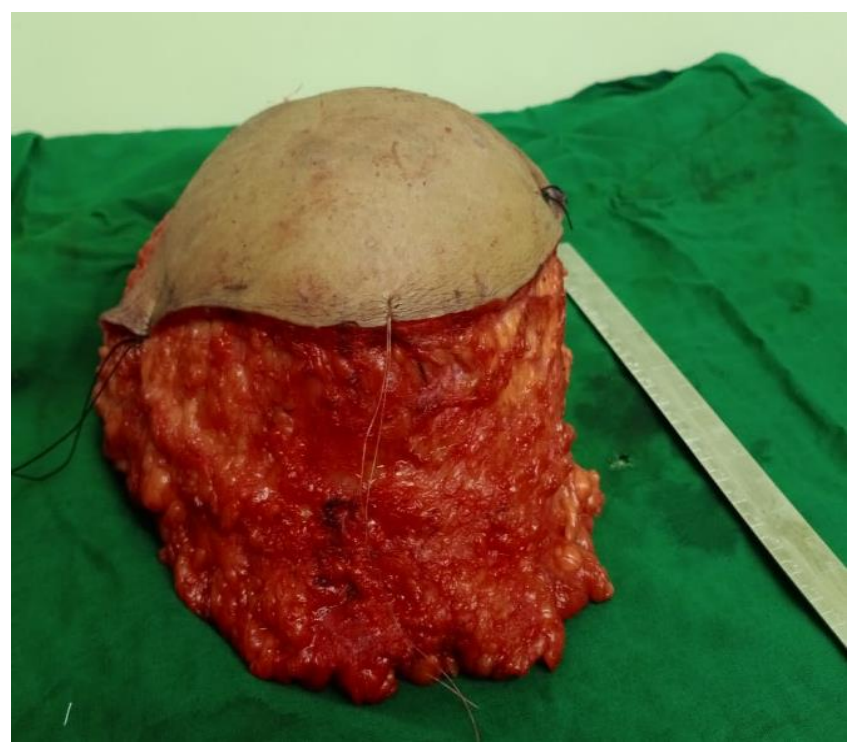

Figure 4: Lateral view of the excised mass of the depth of involvement of the tumour. 


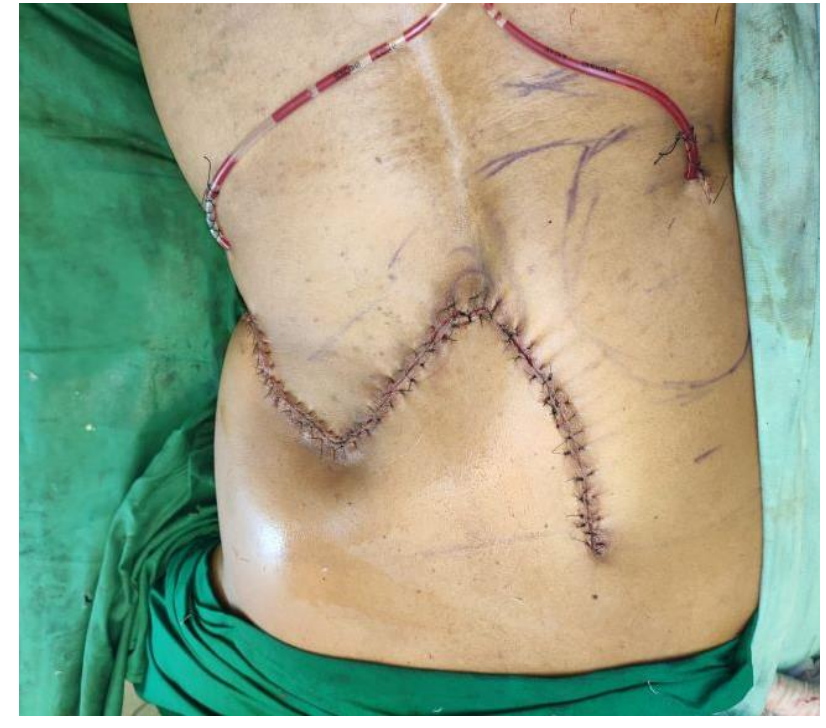

Figure 5: Wide local excision of the mass with $\mathrm{O}$ to $\mathrm{Z}$ double rotational flap with suction drain placement.

The large defect which was left behind following the excision of the mass was repaired by using rotational flap-an $\mathrm{O}$ to $\mathrm{Z}$ double rotational flap, and suction drain placement for the same.

The mass was then sent for histopathological evaluation which showed small round cells which was consistent with the FNAC findings of small round cell tumour. Margins where clear off the disease entity. Histopathology studies also revealed that the Individual tumour cells have scant to vacuolated cytoplasm, round to oval nuclei, thick nuclear membrane with vesicular chromatin and inconspicuous nucleoli.

The patient is to be followed up regularly to prevent recurrence. The data on recurrence rate of the disease is abysmal considering the fact that the number of diagnosed cases in the entire world is less than 60 . Hence regular follow up of this patient as well as every other patient with extra skeletal Ewing's sarcoma needs to be done to study more about the natural history of the disease as well as recurrence rates.

\section{DISCUSSION}

Extra skeletal Ewing's sarcoma is a very rarer clinical entity. The prevalence of an extra skeletal Ewing's sarcoma is merely $1 / 10^{\text {th }}$ of the prevalence of Ewing's sarcoma. The median age of the diagnosis of the patient is usually 20 years with a bimodal peak in distribution of the tumours, the diagnosis being more prevalent in age groups of less than 5 and more than 35 . The diagnosis of such a highly malignant tumour is often missed due to its rare occurrence. However, being highly malignant, the tumour cells disperse, metastasizing, and leading to a very poor 5-year survival rate once the patient gets diagnosed. ${ }^{6}$
Extra skeletal Ewing's sarcoma is now considered in the Ewing's sarcoma group of tumours, due to it being indistinguishable from the latter on microscopy. Ewing's sarcoma group of tumours includes. ${ }^{7}$

Traditional classical Ewing's sarcoma which is confined to the bone and connective tissue, extra skeletal Ewing's sarcoma-histopathology indistinguishable from Ewing's sarcoma and primary neuroectodermal tumours-with neural components more than both extra skeletal and classic Ewing's sarcoma.

Extra skeletal Ewing's sarcoma has a relatively better prognosis as compared to Ewing's sarcoma owing to it superficial occurrence and hence early diagnosis and treatment as compared to any other malignancy in of the Ewing's sarcoma family of tumours.

Histologically, these tumours present in the similar fashion, it is formed by small cells with round hyper chromatic nuclei and a single nucleolus. ${ }^{8}$ The cytoplasm is poorly defined, scarce, of clear coloration and with irregular vacuoles resulting from intracellular deposits of glycogen. ${ }^{9}$

All the tumours which present as small round cell tumour are malignant, the degree of malignancy, that is how aggressive the tumour is depends depending on the mitotic index of the tumour.

As mentioned earlier, in multiple occasions, the family of Ewing's sarcoma like tumours present in the similar manner under the microscope on histopathology. Hence it is pertinent that the sample is also subjected to immunohistochemistry studies as well. The latter helps to differentiate between the multiple differential diagnosis under the broad heading that is small round cell tumour.

The section on further immunohistochemistry the following findings were revealed in this patient.

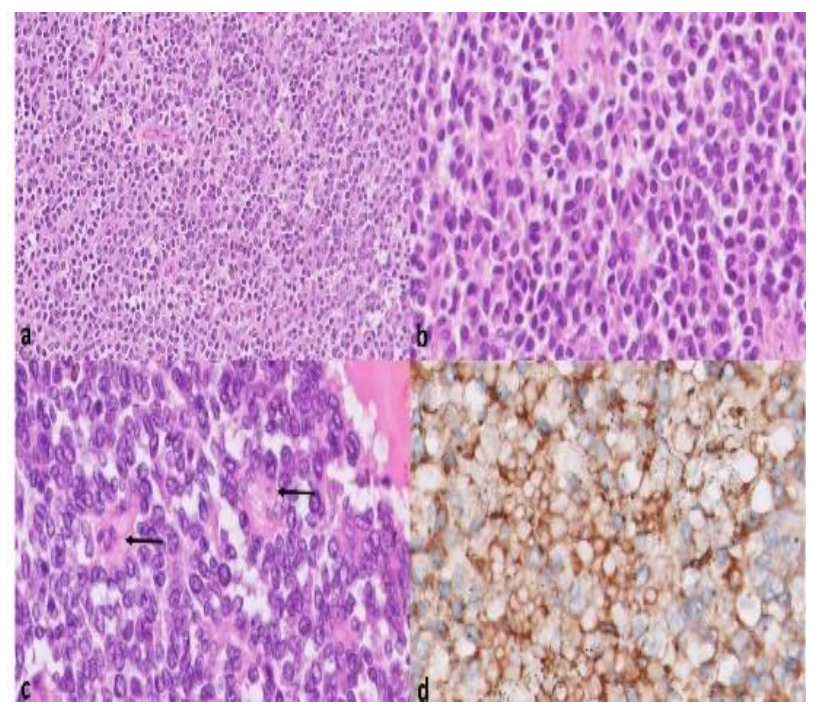

Figure 6: Histopathology and immunohistochemistry section. 
Histopathology showing malignant round cells, tumour cells hyperchromatic nucleus and scant cytoplasm, arrow pointing to rosettes, immunohistochemistry for Mic-2 (CD99) showing diffuse membrane positivity and negative for S100, Desmin, synaptophysin.

Desmin negativity rules out rhabdomyosarcoma, S100, synaptophysin negativity rules out PNET tumours Hence diagnosis of extra skeletal Ewing's sarcoma was made.

Fluorescent in Situ hybridization technique is the final confirmatory test and is ideally recommended in developed countries where the provision is available

Cutaneous and subcutaneous Ewing's sarcoma has better prognosis as compared to skeletal or soft tissue Ewing's sarcoma, owing probably due to earlier diagnosis due to superficial location of the tumour

The treatment of extra skeletal Ewing's sarcoma is mostly surgical, with wide local excision (margin yet to be standardized). ${ }^{10}$ Some articles do advocate for the need for adjuvant chemotherapy, but the matter is still under study. ${ }^{11}$ Radiation therapy is effective in unrespectable diseases. $^{12}$

Our patient is set to undergo an FDG PET scan to check for metastasis or any microscopic tumour deposits. Following which after complete healing of the suture line, the patient is set to undergo chemotherapy cycles composing of vincristine, doxorubicin, cyclophosphamide and actinomycin D, alternating with ifosfamide and etoposide. ${ }^{13}$

\section{CONCLUSION}

Primary extra skeletal Ewing's sarcoma is a very rare clinical entity. Being such a rare diagnosis, the treatment protocol still requires standardization. As what have been observed in our case, a wide local excision keeping a margin of $1 \mathrm{~cm}$, followed by chemotherapy, irrespective of the margins being clear and no evidence of metastasis even on the most advanced PET scan (available in India), as the propensity for micro metastasis is very high.

A quick diagnosis and having no confusion regarding the treatment protocol is necessary for such a highly malignant tumour with tendency to metastasize. Also, a high index of suspicion is also necessary, as this condition can be misdiagnosed as something as simple as a lipoma especially in rural settings where the advent of immunohistochemistry is not available. Immunohistochemistry, is again a very valuable tool required for the final diagnosis of an extra skeletal Ewing's sarcoma.

Funding: No funding sources Conflict of interest: None declared

Ethical approval: Not required

\section{REFERENCES}

1. Yagnik VD, Dawka S. Extra skeletal Ewing's sarcoma/peripheral primitive neuroectodermal tumor of the small bowel presenting with gastrointestinal perforation. Clin Exp Gastroenterol. 2019;12:279-85.

2. Sumegi J, Nishio J, Nelson M, Frayer RW, Perry D, Bridge JA. A novel t $(4 ; 22)$ (q31; q12) produces an EWSR1-SMARCA5 fusion in extra skeletal Ewing sarcoma/primitive neuroectodermal tumor. Mod Pathol. 2011;24(3):333-42.

3. Hübner G. Zur Feinstruktur von formalinfixiertem Biopsie- und Autopsiematerial nach Paraffineinbettung. Virchows Arch Abt A Path Anat. 1970;351:155-67.

4. Dangoor A, Seddon B, Gerrand C. UK guidelines for the management of soft tissue sarcomas. Clin Sarcoma Res 6, $20 \quad$ (2016). https://doi.org/10.1186/s13569-016-0060-4.

5. Javery O, Krajewski K, O'Regan K. A to Z of Extra skeletal Ewing Sarcoma Family of Tumours in Adults: Imaging Features of Primary Disease, Metastatic Patterns, and Treatment Responses. AJR. 2011;197:1015-22.

6. Ahmad R, Mayol BR, Davis M, Rougraff BT. Extra skeletal Ewing's sarcoma. Cancer. 1999;85(3):725-31.

7. Srivastava S, Arora J, Parakh A, Goel RK. Primary extra skeletal Ewing's sarcoma/primitive neuroectodermal tumor of breast. Indian $\mathrm{J}$ Radiol Imaging. 2016;26(2):226-30.

8. Angervall L, Enzinger FM, Angervall L, Enzinger FM. Extraskeletal neoplasm resembling Ewing's sarcoma. Cancer. 1975;36:240-51.

9. Guiter G, Gamboni M, Zakowsk M. The Cytology of Extra skeletal Ewing Sarcoma. Am Cancer Society. 1999;87:141-8.

10. Xie CF, Liu MZ, Xi M. Extraskeletal Ewing's sarcoma: a report of 18 cases and literature review. Chin J Cancer. 2010;29(4):420-4.

11. Hesla,AC.; Papakonstantinou, A.; Tsagkozis, P. Current Status of Management and Outcome for Patients with Ewing Sarcoma. Cancers 2021, 13, 1202. https://doi.org/10.3390/ cancers13061202.

12. Grier H, Krailo M, Tarbell N. Addition of Ifosfamide and Etoposide to Standard Chemotherapy for Ewing's Sarcoma and Primitive Neuroectodermal Tumor of Bone. N Eng J Med. 2013;348:694-701.

13. Dunst $\mathbf{J}$ and Schuck A: Role of radiotherapy in Ewing tumors. Pediatr Blood Cancer. 2004;42:465-70.

Cite this article as: Ahire PP, Nichat PD, Ansari KF, Vismaya KB, Parmar K, Mohanraj A, et al. Primary subcutaneous extra skeletal Ewing's sarcoma: a case report on a very rare clinical entity. Int Surg J 2021;8:2852-2855. 